
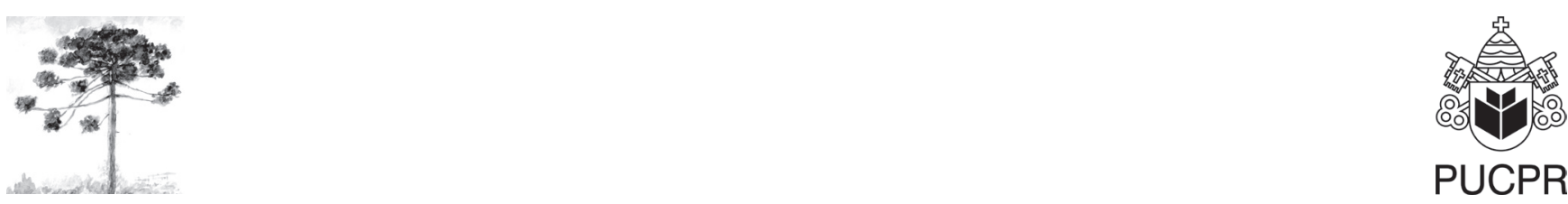

\title{
OCORRENCIA DE Stercorarius parasiticus (LINNAEUS 1758, CHARADRIIFORMES: STERCORARIIDAE) NA ILHA COMPRIDA, LITORAL SUL DE SÃO PAULO
}

\author{
Occurrence of Stercorarius Parasiticus (Linnaeus 1758, Charadriiformes: \\ Stercorariidae) on Ilha Comprida, South Coast of São Paulo State, Brazil
}

Edison Barbieri $^{[a]}$, Celso Alberto Gonçalves ${ }^{[b]}$, Luiz Francisco da Silveira ${ }^{[c]}$

[a] Professor e Pesquisador do Instituto de Pesca/Agência Paulista de Tecnologia do Agronegócio Paulista/APTA, Secretaria de Agricultura e Abastecimento do Estado de São Paulo, Cananeia, SP - Brasil, e-mail: edisonbarbieri@yahoo.com.br

[b] Coordenadoria de Defesa Agropecuária/Secretaria de Agricultura e Abastecimento do Estado de São Paulo, Campinas, SP Brasil, e-mail: celago@terra.com.br

[c] Serviço de Vigilância Agropecuária Internacional, Ministério da Agricultura, Pecuária e Abastecimento, Campinas, SP - Brasil, e-mail: luiver@terra.com.br

\section{Resumo}

Muitas aves marinhas Nearticas migram para a América do Sul para as regiões de invernada, utilizando várias rotas e pontos de paradas ao longo da costa do Oceano Atlântico. A Stercorarius parasiticus é uma ave marinha largamente distribuida por todos os oceanos. É uma ave que migra após a reprodução em direção a América do Sul. Entretanto, há poucas informações sobre a ocorrência de Stercorarius parasiticus ao longo da Costa do Estado de São Paulo. Publicações anteriores a respeito da avifauna do estuário de Iguape, Cananéia, Ilha Comprida não fazem menção à presença da Stercorarius parasiticus na área. Dentro deste contexto, faz-se importante descrever novos registros de sua ocorrência no Estado de São Paulo, uma vez que as mesmas são raras. Durante os trabalhos de amostragem da avifauna, desenvolvidos desde 1998 na Ilha Comprida $\left(24^{\circ} 40^{` 3} 3.16^{\prime} \mathrm{S} 47^{\circ} 25^{`} 47.88^{\prime} \mathrm{W}\right.$ e $\left.24^{\prime} 40^{`} 31.34^{\prime} \mathrm{S} 47^{\circ} 26 ` 13.68^{\prime}\right)$, foram feitas avistagens de Stercorarius parasiticus em apenas uma oportunidade, no mês de outubro de 2008, pousada na praia.

Palavras-chave: Ave Marinha. Migração. Ilha Comprida. São Paulo. Stercorarius parasiticus.

\section{Abstract}

Many Neartic seabirds migrate to South America for the non-breeding season, using several key stopover and wintering sites along the Atlantic coast. The Stercorarius parasiticus is a widely distributed seabird found in every Ocean. It is a migratory bird from the northern region of North 
America that flies southwards. However, there is little information about Stercorarius parasiticus occurrence of along the São Paulo State coast. Former publications concerning the birds of IguapeIlha Comprida estuary did not mention the presence of this bird in the region. Thus it is important that forthcoming appearances be recorded. Throughout the bird-sample selection stage, made since 1998 at Iguape - Ilha Comprida estuary (24 $43^{\prime} 09.99^{\prime \prime} S$ and $\left.47^{\circ} 29^{\prime} 50.79^{\prime \prime} \mathrm{W}\right)$ - $200 \mathrm{~km}$ from São Paulo -, the bird was seen in October (2009), resting at Ilha Comprida beach. This note reports the occurrence of Stercorarius parasiticus in Ilha Comprida, south coastal of São Paulo State, Brazil.

Keywords: Seabird. Migration. Ilha Comprida. São Paulo. Stercorarius parasiticus.

\section{INTRODUÇÃO}

Stercorarius parasiticus é uma ave Caradriiforme, da família Stercorariidae. S. parasiticusé semelhante aos outros Stercorariidae, que frequentemente perseguem outras aves marinhas para lhes roubar o alimento, sendo, assim, cleptoparasitas. Esta espécie nidifica na Escócia, na Escandinávia e na Islândia e inverna no Oceano Atlântico (1).

Tal como as outras espécies de Stercorariidae, de menores dimensões, esta é mais facilmente distinguível quando com plumagem de adulto nupcial, com as penas centrais da cauda afiladas, como característica mais marcante $(1,2)$. No entanto, as plumagens de imaturos e juvenis são extremamente difíceis de separar dos congêneres Stercorarius pomarinus e Stercorarius longicaudus (3). Neste caso, a forma do corpo e das asas é a melhor ajuda. $S$. parasiticus tem o corpo menor que o $S$. pomarinus, tendo maior projeção do peito e bico mais curto $(1,2)$. Este último é praticamente uniforme em termos de coloração, contrastando com o bico bicolor dos seus congêneres. Tal como as outras espécies, possui um barrete escuro que contrasta com o claro do pescoço, no caso dos adultos (1). Possui um voo ágil, com batimentos amplos, que lhe permite perseguir algumas das aves marinhas mais bem adaptadas ao voo rápido. O $S$. parasiticus ocorre em variações de plumagem que podem ir do totalmente escuro a indivíduos claros, com o pescoço amarelado, com todas as plumagens intermédias possíveis.

S. parasiticus é uma migrante trans-equatorial que inverna na costa sul-americana (4, 5, 2, 6-9). Segundo Sick (2), a espécie é um visitante regular da costa brasileira, registrando a captura de um indivíduo na Ilha do Governador, no Estado do Rio de Janeiro, em 1970. Olmos (8), baseado nos arquivos do BTO, menciona o registro de $S$. parasiticus para o Estado do Espírito Santo, em Itaúnas (1992) e Guarapari (1995), e em Maceió, no Estado de Alagoas (1965).
A costa do Uruguai e da Argentina se constitui num dos principais destinos de $S$. parasiticus (6). Segundo Belton (10), S. parasiticus tem sua presença registrada como pouco frequente no Rio Grande do Sul, entretanto, para Veit (5), essa espécie tem tido presença regular nesse Estado. No litoral do Paraná foi registrada por Barnett et al. (9) e em Santa Catarina, por Branco et al. (11). Já em São Paulo, Pinto (1964) fez o primeiro registro, seguido mais recentemente por Olmos (3).

Em 9 de outubro de 2008 observamos um exemplar na praia da Ilha Comprida (24 43'09,99”S $47^{\circ} 29^{\prime}$ 50,79' W), São Paulo, o qual foi identificado como $S$. parasiticus. Duas fotos desse exemplar (Figura 1) mostram um $S$. parasiticus, com o bico escuro, tarsos mais claros que os pés e retrizes centrais muito alongadas e com pontas afiladas, e que se mostrou bastante manso.

Baseando-se nessas características, o referido exemplar foi identificado como $S$. parasiticus. A faixa peitoral incompleta sugere tratar-se de um jovem. O $S$. parasiticus ocorre em variações de plumagens que podem variar da tonalidade totalmente escura, a indivíduos claros, com os pescoços amarelados, com todas as plumagens intermédias possíveis (2). Este é o segundo registro da espécie para a Ilha Comprida e para o Estado de São Paulo. Sendo relevante salientar que no trabalho de Barbieri e Tavares (12) sobre as aves da Ilha Comprida nenhum exemplar dessa espécie foi registrado.

S. parasiticus tem sua presença sido registrada ocasionalmente no Rio Grande do Sul (5), Santa Catarina (11) e Paraná (9). Entretanto, ainda faltam trabalhos sistemáticos e mais aprofundados para todo o litoral brasileiro e, principalmente, para o litoral de São Paulo. Tais trabalhos seriam de grande importância, pois avaliariam os possíveis registros da espécie, analisariam se tais ocorrências são esporádicas ou sazonais, com que frequência a espécie visita o Estado de São Paulo e estimaria o tamanho da população visitante. 


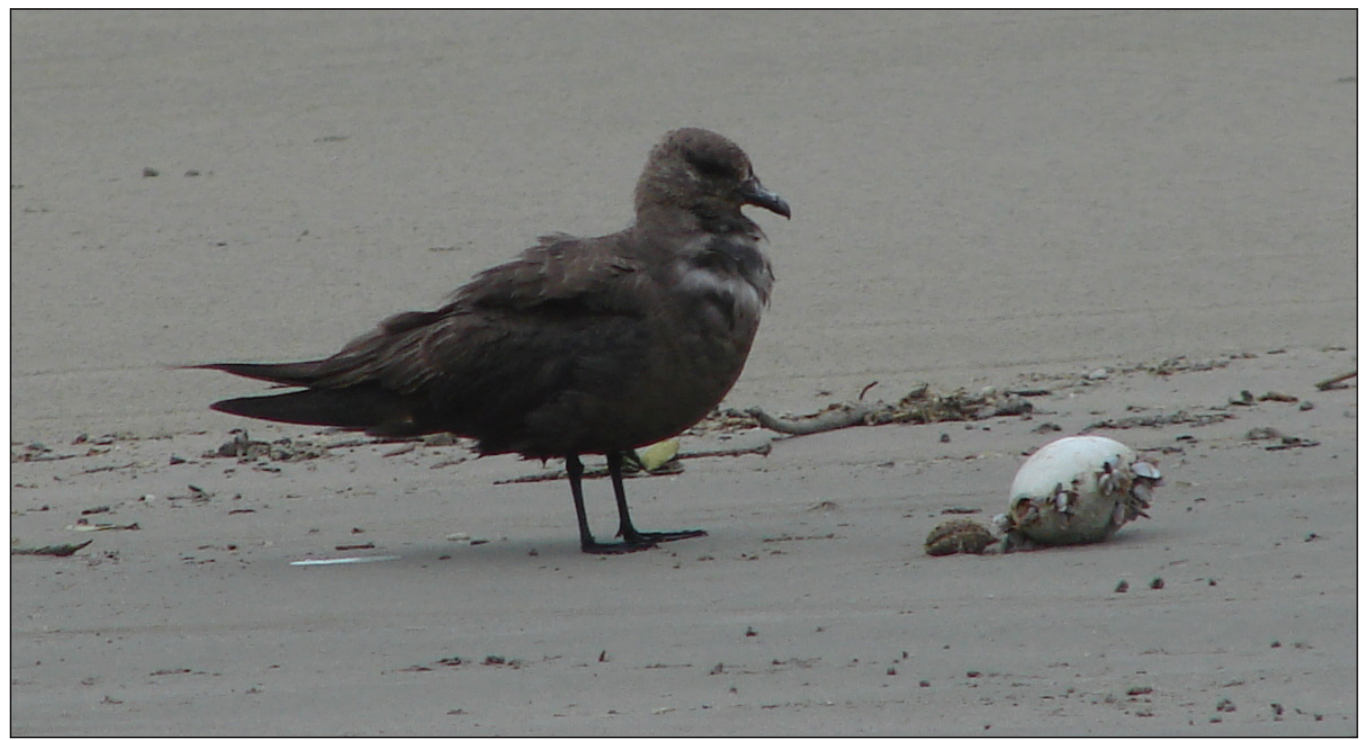

FIGURA 1 - Stercorarius parasiticus na Praia da Ilha Comprida (Boqueirão Norte), São Paulo Fonte: Edison Barbieri.

\section{AGRADECIMENTOS}

Agradecemos ao Instituto de Pesca e à Coordenadoria de Defesa Agropecuária, da Secretaria da Agricultura do Estado de São Paulo, e ao Ministério da Agricultura, Pecuária e Abastecimento, pelo apoio para a realização do presente trabalho.

\section{REFERÊNCIAS}

1. Harrison, P. Seabirds: an identification guide. London \& Sidney: Croom Helm; 1983.

2. Sick, H. Ornitologia Brasileira. Rio de Janeiro. Nova Fronteira; 1997.

3. Olmos, F. Revisão dos registros de Stercorarius pomarinus no Brasil, com notas sobre registros de $S$. longicaudus e $S$. parasiticus (Charadriiformes: Stercorariidae). Nattereria. 2000;1:29-33.

4. Borges SH. Primeiro registro de Myrmeciza disjuncta para o Brasil (Passeriformes: Thamnophilidae). Nattereria. 2000;1. [acesso em 12 jun. 2006]. Disponível em: http://www.cbro.org.br/CBRO/pdf/ nat1 not1.pdf.

5. Veit, RR. Long-tailed jaegers wintering along the Falkland current. American Birds. 1995;39(5):873-8.

6. Vooren CM, Chiaradia A. Stercorarius longicaudus and S. parasiticus in southern Brazil. Ardea, 1989;77:233-5.
7. Olsen K M, Larsson H. Skuas and jaegers: a guide to the skuas and jaegers of the world. New Haven: Yale University Press; 1997.

8. Olmos, F. Non-breeding seabirds in Brasil: a review of band recoveries. Ararajuba. 2002;10(1):31-42.

9. Barnett, JM, Minns J, Kirwan GM, Remold H. Informações adicionais sobre as aves dos estados do Paraná, Santa Catarina e Rio Grande do Sul. Ararajuba. 2002;12(1):53-6.

10. Belton, W. Aves do Rio Grande do Sul: distribuição e biologia. São Leopoldo: Ed. Unisinos; 1994.

11. Branco, JO, Fracasso HAA, Verani JR. Interações entre aves marinhas e a pesca de camarões na Armação do Itapocoroy, Penha, SC. In: Branco JO, Marenzi, AWC. (Org.). Bases ecológicas para um desenvolvimento sustentável: estudos de caso em Penha, SC. Itajaí, SC: UNIVALI, 2006. p. 171-182.

12. Barbieri, E, Paes, ET. The birds at Ilha Comprida beach (São Paulo state, Brazil): a multivariate approach. Biota Neotropical. 2008. [acesso em 10 jun. 2006 8(3): http://www.biotaneotropica.org.br/ v8n3/en/abstract?article+bn00408032008.

Recebido: 09/07/2007 Received: 07/09/2007

Aprovado: 28/10/2007 Approved: 10/28/2007 